\title{
Cellular mechanisms of MR regulation of adipose tissue physiology and pathophysiology
}

\author{
Andrea Armani', Vincenzo Marzolla', Andrea Fabbri ${ }^{2}$ and Massimiliano Caprio ${ }^{1,3}$ \\ 'Laboratory of Cardiovascular Endocrinology, IRCCS San Raffaele Pisana, Via di Val Cannuta, 247, Rome, Italy \\ ${ }^{2}$ Endocrinology Unit, Department of Systems Medicine, S. Eugenio and CTO A. Alesini Hospitals, \\ University Tor Vergata, Rome, Italy \\ ${ }^{3}$ University San Raffaele, Rome, Italy
}

Correspondence should be addressed to M Caprio Email massimiliano.caprio@ sanraffaele.it

\begin{abstract}
In addition to the well-documented expression and activity of the mineralocorticoid receptor (MR) in the kidney, in the last decade research on MR has also revealed its important role in regulating functions of extrarenal tissues, including adipose tissue, where MR is involved in adipocyte fundamental processes such as differentiation, autophagy and adipokine secretion. MR expression is increased in adipose tissue of murine models of obesity and in obese human subjects, suggesting that over-activation of the mineralocorticoid signaling leads to dysfunctional adipocyte and associated metabolic disorders. Notably, pharmacological blockade of MR prevents metabolic dysfunctions observed in obese mice and suggests a potential therapeutic use of MR antagonists in the treatment of obesity and metabolic syndrome. However, the molecular pathways affected by MR blockade have been poorly investigated. This review summarizes the functions of MR in the adipocyte, discusses potential signaling pathways mediating MR action, and describes post-translational modifications regulating its activity.
\end{abstract}

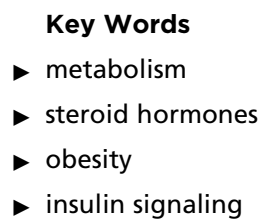

Journal of Molecular Endocrinology (2015) 55, R1-R10

\section{Introduction}

Mineralocorticoid receptor (MR) activation by its primary ligand aldosterone is a well-known mechanism acting at the renal level as a regulator of plasma volume, salt homeostasis and blood pressure (Funder 2005). Importantly, besides aldosterone, MR can bind glucocorticoids with a higher affinity (10-fold) than the glucocorticoid receptor (GR) (Reul \& De Kloet 1985). In epithelial tissues, the enzyme 11beta hydroxysteroid dehydrogenase type II (11ßHSD2), which converts active glucocorticoids into inactive metabolites, results in aldosterone selectivity of MR and excludes its activation by glucocorticoids (Funder 2009). On the other hand, in non-epithelial tissues such as the brain (De Kloet et al. 2000), cardiomyocytes (Farman \& Bocchi
2000) and adipose tissue (Rondinone et al. 1993, Caprio et al. 2007) either low levels or lack of 11ßHSD2 lead to prevailing occupancy of MR by glucocorticoids (Funder 2000). In the recent years, the identification of MR in different non-epithelial tissues has led to the development of a novel field of research whose aim is to investigate the extra-renal functions of MR. Indeed, distribution of MR in such a wide range of tissues suggested novel unexpected roles for MR, in diverse physiological and pathophysiological contexts, including metabolic syndrome, oxidative stress, stress adaptability, inflammation and more.

In this context, several research groups identified the adipocyte as a novel cellular model for investigating the

Published by Bioscientifica Ltd 
function of MR in the pathophysiology of obesity and metabolic syndrome. Either knockdown or pharmacological blockade of MR inhibits adipogenesis in 3T3L1 cultures (Caprio et al. 2007, 2011), revealing an important role for this transcription factor in adipose tissue physiology and pathophysiology. Pharmacological MR antagonism has been shown to counteract dysfunctional metabolism of adipose tissue (Guo et al. 2008, Hirata et al. 2009, Wada et al. 2010). Remarkably, conversion of white into brown adipose tissue, a process induced by MR blockade, takes part in the anti-obesity effects of MR antagonism (Armani et al. 2014a).

However, most of the signaling pathways downstream of MR activation by aldosterone or glucocorticoids in the adipocyte still remain unclear.

This review highlights the state of the art in the field about the role of MR activation in adipose tissue, under physiologic and pathophysiologic conditions, and briefly describes potential signaling pathways of the adipocyte which could be targeted by MR. Finally, we discuss the potential modulation of MR activity in the adipocyte by post-translational modifications, which are known to influence MR function, hence potentially affecting the physiology of adipose tissue.

\section{Role of MR in adipocyte function}

MR expression and function has been extensively studied in adipocyte cultures as well as in the fat of murine models of obesity, by our laboratory and other groups (Zennaro et al. 1998, Caprio et al. 2007, Guo et al. 2008, Hirata et al. 2009, Armani et al. 2014a). MR activation upregulates the expression of adipocyte markers and promotes adipogenesis in 3T3L1 adipose cells (Caprio et al. 2007). Conversely, pharmacological blockade of MR reduces adipocyte differentiation in 3T3L1 cells as well as in primary human adipocytes (Caprio et al. 2011). Consistent with these data, murine primary adipocyte cultures prepared from MR knockout mice show defective adipogenesis (Hoppmann et al. 2010) and knockdown of MR in human primary adipocyte cultures blocks adipogenesis, confirming that MR plays a relevant role both in mouse and human adipogenesis (Hoppmann et al. 2010, Armani et al. 2014b). Altogether, these studies in vitro suggest that impaired activity of MR inhibits adipocyte differentiation. In vivo studies in obese mouse models show increased levels of MR transcript in adipose tissue in comparison with lean mice, suggesting enhanced activation of this receptor (Hirata et al. 2009). Treatment of obese mice with MR antagonists counters fat mass gain
(Wada et al. 2010, Armani et al. 2014a) as well as altered expression of adiponectin, PPAR $\gamma$ and leptin (Guo et al. 2008, Hirata et al. 2009), confirming that MR activity promotes expression of adipocyte genes and regulates adipose tissue function.

Notably, in obese mice, the increased transcript expression of cytokines tumor necrosis factor alpha (TNF $\alpha$ ), monocyte chemoattractant protein-1 (MCP-1), interleukin-6 (IL-6) (Guo et al. 2008, Hirata et al. 2009), macrophage markers CD68 and CD11 as well as the increased presence of crown-like structures associated with enhanced inflammation and adipocyte apoptosis (Murano et al. 2008), are prevented by treatment with MR antagonist, indicating that adipocyte-specific MR activity affects the expression of pro-inflammatory adipokines and macrophage recruitment and plays a crucial role in generating the chronic inflammatory profile observed in obesity (Guo et al. 2008, Hirata et al. 2009). Production of reactive oxygen species (ROS) is higher in adipose tissue of obese mice and increased levels of ROS in adipose depots have been shown to cause metabolic dysfunction and accumulation of fat (Furukawa et al. 2004). Interestingly, altered expression of NADPH oxidase subunits, involved in ROS production, is prevented by MR antagonism, indicating an additional protective effect of MR blockade on adipocyte function (Hirata et al. 2009). However, $\mathrm{H}_{2} \mathrm{O}_{2}$ treatment of 3T3L1 adipocytes (Hirata et al. 2009) increases, by itself, MR transcript levels, suggesting that MR activity is amplified by oxidative stress and plays a role in reinforcing ROS production in the dysfunctional adipocyte.

Although these studies demonstrate that enhanced MR activity contributes to the dysregulation of adipocyte function, a recent study by Kuhn et al. (2014) found that transgenic mice globally overexpressing human MR, in comparison with WT mice, display resistance to highfat-diet-induced weight gain, protection from fat mass expansion and impaired glucose tolerance, thus showing beneficial metabolic effects similar to those observed in obese mice treated with MR antagonists (Hirata et al. 2009, Wada et al. 2010, Armani et al. 2014a). These results may appear contradictory to the current understanding of adipocyte MR, but the study mentioned above is focused on a different model in which global overexpression of MR triggers an integrated adaptive response. Interestingly, adipose tissue of these transgenic mice showed decreased adipocyte size as compared to WT mice, suggesting that MR overactivation might inhibit adipocyte maturation, resulting in unexpected effects on adipogenesis. However, the authors showed that adipocyte cultures from mice

Published by Bioscientifica Ltd. 
overexpressing MR did not present defective adipogenesis, excluding the possibility that adipocyte MR overexpression may lead to intrinsic defects in adipocyte differentiation. On the other hand, conditioned media from cultures of MR overexpressing macrophages impaired in vitro adipogenesis, suggesting that secretory molecules released from macrophages with enhanced MR activity may impair adipogenesis also in vivo. Notably, together with the effects on adipocyte maturation, changes in M1 (pro-inflammatory)/M2 (anti-inflammatory) polarization of the MR-overexpressing macrophages, were observed in these transgenic mice, raising the possibility of a causal association between macrophage polarization and extent of adipocyte differentiation.

MR activity in the macrophage is known to influence its polarization (Usher et al. 2010). Interestingly, mice with macrophage-specific deletion of the MR gene display increased M2 polarization and cardio-protective effects; however, the metabolic profile and adipose tissue function of these mice has still not been investigated (Rickard et al. 2009, Usher et al. 2010). Indeed, as discussed by Marzolla et al. (2014), regulation of macrophage polarization by MR might have a role in regulating development, inflammatory state and insulin sensitivity of adipose tissue.

\section{Role of MR in 'browning' of white adipose tissue}

In rodents and newborn humans, white and brown adipose tissue (WAT and BAT, respectively) represent two distinct types of adipose tissue with different morphologies and functions (Cinti 2012). Whereas WAT is deputed to store energy in the form of triglycerides, the BAT function is to burn fat and convert chemical energy into heat for thermogenesis (Seale et al. 2009, Cinti 2012).

In addition to classical BAT, recent studies have discovered in mouse WAT the presence of inducible brown-like adipocytes termed 'beige' or 'brite' (brown in white) (Kajimura et al. 2010). These cells appear in WAT of mice exposed to cold or treated with $\beta 3$-adrenergic agonists (Kajimura et al. 2010). The beige adipocytes have morphology with multilocular lipid-droplet and a high content of mitochondria and express a number of genes, including UCP1, Cidea and PGC1 $\alpha$, that are known to be expressed in BAT adipocytes (Harms \& Seale 2013). Notably, although the beige adipocytes display thermogenic activity, these cells do not originate from myogenic factor 5 (myf5)-positive embryonic precursors, from which BAT and skeletal muscle derive, and should be considered as a distinct subtype of adipose cells (Seale et al. 2008).
Interestingly, $\beta$-adrenergic stimulation has been shown to increase UCP1 levels and uncoupled respiration rate of beige adipocytes, leading to the acquisition of brown fat features by WAT (Wu et al. 2012), a process known as 'browning.' However, whether the beige cells represent a specific pool of adipose cells distinct from white adipocytes, or derive by conversion of mature white adipocytes to brown-like adipose cells, still remains unclear (Rosenwald et al. 2013). A number of studies shows that browning protects mice from weigh gain, fat mass expansion and associated dysregulation of glucose and lipid metabolism (Harms \& Seale 2013). Importantly, recent data have suggested that brown adipocytes can be detected also in adult human subjects and its amount is inversely correlated with BMI, suggesting a potential protection against obesity (Wu et al. 2012, 2013). Therefore, induction of browning by appropriate pharmacological treatment may represent an innovative approach to treat obesity and metabolic syndrome. In this context, a recent study by our laboratory has shown that treatment of mice fed a high-fat-diet (HFD) with MR antagonists induces browntypical features in WAT and confers protection against obesity and impaired glucose tolerance (Armani et al. 2014a). Increased expression of brown adipocyte-specific genes, including UCP1, was detected both in white fat depots from mice and in murine primary adipocyte cultures treated with MR antagonists. These data are consistent with findings from previous studies that showed a reduction of UCP1 transcripts in brown adipocyte cell line T37i treated with aldosterone (Viengchareun et al. 2001).

Importantly, our study shows that MR blockade promotes brown fat marker gene expression in white adipocyte precursor cells and concomitantly attenuates the levels of transcripts highly expressed in the white adipocyte, suggesting opposite effects of MR antagonism on white and brown adipogenesis (Armani et al. 2014a). However, there is still no evidence that MR may exert opposing effects on WAT- and BAT-specific gene expression through direct action on regulatory elements. Time-course analysis of gene expression might provide some hints about mechanisms of gene regulation mediated by MR. We speculate that MR signaling pathways in the adipocyte might modulate the expression of transcription factor(s) or transcriptional coregulators affecting adipocyte gene transcription profiles (Fig. 1).

Clearly, further studies are needed to investigate the molecular mechanisms by which MR affects the transcriptional program driving white and brown adipocyte differentiation.

Published by Bioscientifica Ltd 


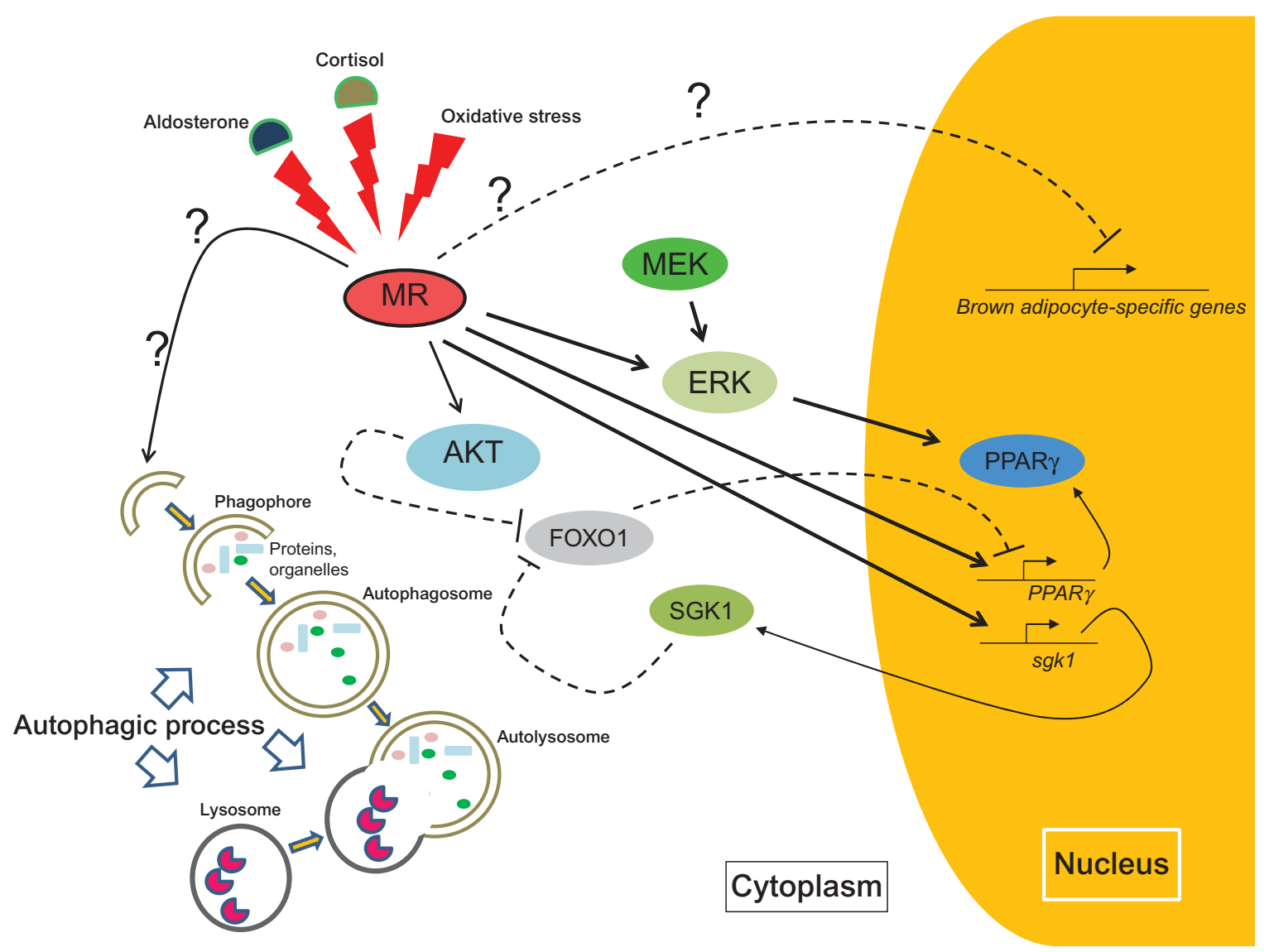

\section{Figure 1}

MR regulates adipogenesis-linked signaling pathways. In the adipocyte, MR activation by either aldosterone, cortisol or oxidative stress results in direct and non-direct transcriptional effects. MR activity promotes the autophagic flux and adipocyte differentiation. MR blockade has been shown to induce brown adipocyte-specific genes; MR induces transcription of PPAR $\gamma$, the master regulator of adipocyte differentiation, and sgk1.

\section{Regulation of autophagy by MR in the adipocyte}

Autophagy is a process allowing eukaryotic cells to regulate organelle and protein turnover and maintain tissue homeostasis (Mizushima \& Levine 2010). The autophagic process consists in a complex sequence of subsequent steps beginning with the formation of the 'phagophore,' which expands and sequesters organelles and proteins in a doublemembrane autophagosome. Fusion of the autophagosome with the lysosome leads to degradation of the contents of the autophagosome; the resulting degradation products move out to the cytoplasm where they can be used to build proteins and macromolecules (Mizushima 2007, Glick et al. 2010). Increased autophagosome abundance has been observed during 3T3L1 preadipocyte differentiation and enhanced autophagic flux has been detected in primary
SGK1 has been shown to phosphorylate and prevent nuclear translocation of FOXO1 and its anti-adipogenic function. Phosphorylation of FOXO1 by AKT also leads to block FOXO1 inhibitory effect on PPAR $\gamma$ expression. Indirect actions of MR include activation of AKT and ERK. Both of these protein kinases are required for adipocyte differentiation and may mediate the pro-adipogenic effects of MR.

mouse embryonic fibroblasts (MEFs) induced to differentiate into adipocytes (Novikoff et al. 1980, Baerga et al. 2009). Among the autophagy-related genes (atg) identified in mammals, atg7 and atg5 roles have been studied both in vivo and in cell culture (Singh et al. 2009, Baerga et al. 2009). atg7 functions as an E1 ubiquitin activating enzyme for $\operatorname{atg} 12$ conjugation to $\operatorname{atg} 5$; $\operatorname{atg} 5-\operatorname{atg} 12$ conjugate formation is required for autophagosome formation (Glick et al. 2010).

Notably, knockdown of atg7 in 3T3L1 adipocytes represses differentiation (Singh et al. 2009) and atg5-/MEFs show defective adipogenesis, altogether indicating a pivotal role of autophagy in adipocyte differentiation (Baerga et al. 2009).

Adipocyte-specific atg7-knockout mice have been explored in two distinct studies, in order to investigate

Published by Bioscientifica Ltd. 
the effects of impaired autophagy in adipose tissue. These models show resistance against HFD-induced obesity and display improved insulin sensitivity (Singh et al. 2009, Zhang et al. 2009). Interestingly, Singh et al. show that these transgenic mice have reduced WAT size and an increased amount of interscapular BAT. Moreover, WAT in these knockout mice display histological features of BAT: smaller adipocyte size, adipocytes with multilocular lipid droplets and higher number of mitochondria. Moreover, WAT showed increased protein levels of uncoupling protein 1 (UCP1) and PPAR $\gamma$ coactivator 1alpha (PGC1 $\alpha$ ), a master regulator of mitochondrial biogenesis, and higher abundance of the mitochondrial enzymes cytochrome oxidase and cytochrome $c$. Singh et al. excluded the possibility that the reduced size of WAT could be due to a decreased number of adipocyte precursors whose levels were not changed in the transgenic mice, and suggested that the effects of impaired autophagy occurred after the development of white adipose cells through transdifferentiation of mature white into brown adipocytes.

Notably, although Zhang et al. observed that WAT of atg7 knockout mice acquires morphological features of BAT, no changes in brown adipocyte markers were detected, suggesting that autophagy inhibition in this study has beneficial effects on adipose tissue and glucose metabolism even in the absence of conversion of white adipocytes into thermogenic brown adipocytes.

Interestingly, autophagy not only affects WAT differentiation and metabolism, but also classical BAT development. BAT derives from myogenic factor 5-positive (myf5) progenitors. Transgenic mice lacking atg7 in myf5+ progenitors show loss of autophagy in BAT that displays impaired differentiation (Martinez-Lopez et al. 2013).

A recent study from our laboratory has shown that MR activity modulates autophagic flux in adipose cell cultures (Armani et al. 2014a). In 3T3L1 cells and primary murine adipocytes, MR activation by aldosterone treatment increases autophagy, whereas MR blockade reduces it (Fig. 1). In mice fed HFD, pharmacological blockade of MR reduces adipose tissue autophagic flux and, in turn, induces browning of WAT, confirming the effects of impaired autophagy observed in WAT of atg7 knockout mice (Armani et al. 2014a).

Importantly, autophagy is increased in adipose tissue of obese humans and correlates with the degree of visceral adiposity and adipocyte hypetrophy, and elevation of autophagic rate has been detected in adipose tissue of subjects with insulin resistance (Kovsan et al. 2011).

All these data suggest that dysregulation of autophagy may lead to dysfunctional adipocytes. Indeed, the ability of MR to modulate adipocyte autophagic flux confirms the key role of this transcription factor in regulating adipose tissue function.

\section{Role of MR in regulating adipogenesis-linked signaling pathways}

MR regulates adipocyte differentiation and metabolism mostly through regulating gene transcription (Yang \& Young 2009). Such modulation may occur both via direct binding on regulatory regions of target genes or through regulation of protein kinase signaling pathways. During differentiation of 3T3L1 preadipocytes MR activation has been shown to upregulate transcription of adiponectin, leptin, resistin and PPAR $\gamma$ (Caprio et al. 2007). IL-6, TNF $\alpha$ and MCP-1 (Guo et al. 2008) transcripts have been also found increased by aldosterone in terminally differentiated 3T3L1 adipocytes. Inhibition of MR transcriptional activity by the MR antagonist drospirenone (Caprio et al. 2011) reduces transcript levels of the pro-adipogenic genes $\operatorname{PPAR} \gamma$ and CEBP $\alpha$ in human adipocyte cultures (Caprio et al. 2011). However, in vitro studies never explored the molecular mechanisms by which MR activates the transcription of the above-mentioned adipogenic genes, and a direct binding of MR on regulatory regions of these genes has still not been described in the adipocyte.

A consensus glucocorticoid response element (GRE) is located at position $-429 /-414$ in the human serum- and glucocorticoid-regulated kinase 1 (Sgk1) promoter; chromatin immunoprecipitation (ChiP) data have shown that MR directly binds this region of the $S g k 1$ gene and stimulates its transcription in embryonic kidney 293 cells (HEK293) (Lee et al. 2013). SGK1 has been shown to mediate aldosteroneinduced $\mathrm{Na}+$ reabsorption by renal epithelia (McCormick et al. 2005), but a role for $S g k 1$ has been also demonstrated in adipocyte differentiation (Di Pietro et al. 2010). In fact, $S g k 1$ expression increases during adipose differentiation and promotes this process through direct phosphorylation of forkhead box protein O1 (FOXO1) and subsequent exclusion of this transcription factor from the nucleus (Di Pietro et al. 2010). FOXO1 inhibits adipogenesis via reduced expression and transcriptional activity of PPAR $\gamma$; SGK1 prevents the anti-adipogenic activity of FOXO1, counteracting its nuclear localization (Nakae et al. 2003).

In 3T3L1 adipocytes, transcription of $S g k 1$ is induced by glucocorticoids, which are able to stimulate $S g k 1$ expression through MR activation (Di Pietro et al. 2010). Thus, as shown in kidney cells, MR might also directly bind and promote activity of the $S g k 1$ gene promoter in adipose tissue (Fig. 1).

Published by Bioscientifica Ltd. 
The mitogen-activated protein kinase/MEK/ERK pathway has been recently confirmed as a fundamental regulator of adipocyte metabolism (Banks et al. 2015). MEK is a dual threonine and tyrosine recognition kinase that phosphorylates and activates ERKs, a family of serine/threonine kinase, which in turn affects cellular proliferation and differentiation (Boulton \& Cobb 1991, Pearson et al. 2001, Ebisuya et al. 2005). Preadipocytes purified from ERK1 - / - mice display defective adipogenesis, indicating that ERK1 activity is required for adipocyte differentiation (Bost et al. 2005). Notably, adipose tissue shows increased activity of ERK in mice fed HFD and in obese human subjects with type 2 diabetes (Carlson et al. 2003, Bost et al. 2005). At a molecular level, ERK phosphorylates PPAR $\gamma$ at serine 273 (Banks et al. 2015). Such specific phosphorylation of PPAR $\gamma$ has been detected in obese mice, with concomitant reduced expression of adiponectin and adipsin, which in turn favor insulin sensitivity (Banks et al. 2015). Blockade of the phosphorylation at this serine residue by MEK inhibitors results in beneficial effects on adipocyte metabolism. In fact, obese $o b / o b$ mice treated with MEK inhibitor display improved glucose tolerance, increased expression of adiponectin and adipsin, and upregulation of genes involved in the induction of browning of WAT (Banks et al. 2015). In rat renal fibroblasts, activation of MR by aldosterone has been shown to activate ERK (Nagai et al. 2005) and treatment with the MR antagonist eplerenone blocks aldosteroneinduced ERK activation, indicating specific involvement of MR in ERK modulation and suggesting potential regulation of a fundamental metabolic pathway in the adipocyte (Fig. 1).

Among the adipogenic factors commonly used in the induction of adipogenesis in vitro, insulin promotes the adipose conversion process through activation of AKT protein kinase (Cho et al. 2004, Armani et al. 2010). Notably, ectopic expression of activated AKT is able to induce differentiation of 3T3L1 preadipocytes (Gagnon et al. 1999) and, in mice, disruption of insulin signaling in adipose tissue leads to reduced fat mass (Bluher et al. 2002). In human macrophage cell lines, MR activation has been shown to increase AKT activity (Lin et al. 2014). It is tempting to speculate that, if such mechanism occurs also in differentiating preadipocytes, increased AKT activation by MR might display relevant effects on adipogenesis (Fig. 1).

Importantly, aldosterone treatment in vascular smooth muscle cells (VSMCs) has been shown to cause proteosomal degradation of insulin receptor substrate1 (IRS1), a signaling adaptor for insulin (Taniguchi et al.
2006), with subsequent reduction in insulin-induced AKT activation and glucose uptake (Bender et al. 2013). Thus, MR activity might also trigger adverse effects on insulin signaling. In 3T3L1 adipocytes, aldosterone treatment reduces IRS1 protein levels with subsequent impaired insulin-induced glucose uptake. Co-treatment with eplerenone does not change IRS1 protein abundance, whereas the GR antagonist RU486 prevents such degradation, suggesting that MR activity is not responsible for changes in the IRS1 protein (Wada et al. 2009).

Clearly, a deeper understanding of the effects of MR activity on IRS1 protein levels and on insulin signaling in adipose tissue in vivo will require further investigations.

\section{Modulation of MR activity by adipogenic signaling pathways and post-translational modifications}

Modulation of MR activity can occur through posttranslational modification by protein kinases and enzymes involved in adipogenic pathways (Faresse 2014). Among the transcription factors involved in adipogenesis, a relevant role for CREB has been demonstrated. Adipogenesis is promoted by CREB, whose phosphorylation/ activation stimulates expression of adipocyte genes such as phosphoenolpyruvate carboxykinase (PEPCK), fatty acid binding protein (FABP), fatty acid synthase (FAS), CEBP- $\beta$ and $-\delta$ (Reusch et al. 2000). cAMP-dependent protein kinase A (PKA) is able to phosphorylate CREB and, in turn, stimulate adipocyte differentiation (Zhang et al. 2004, Yang et al. 2008, Doorn et al. 2012), although other evidence suggests that PKA activation suppresses adipogenesis (Li et al. 2008).

In HepG2 hepatocytes, PKA has been shown to increase MR binding to promoters containing GRE elements and its transcriptional activity, hence showing synergistic effect with aldosterone (Massaad et al. 1999). Interestingly, phosphorylation of MR by PKA has not been detected in these cells; these data suggest an indirect effect of PKA on MR transactivation, probably through interaction with transcriptional repressors/co-activators of MR. (Massaad et al. 1999). We cannot exclude that similar regulatory mechanism involving these factors might promote MR transactivation and stimulate transcription of MR-induced genes in the adipocyte (Fig. 2).

However, phosphorylation of MR has been detected in renal cells, cardiomyocytes and adipocytes (Galigniana 1998, Desarzens et al. 2014). Aldosterone itself is able to induce phosphorylation of MR on serine residues located in the N-terminal domain with subsequent proteosomal

Published by Bioscientifica Ltd. 


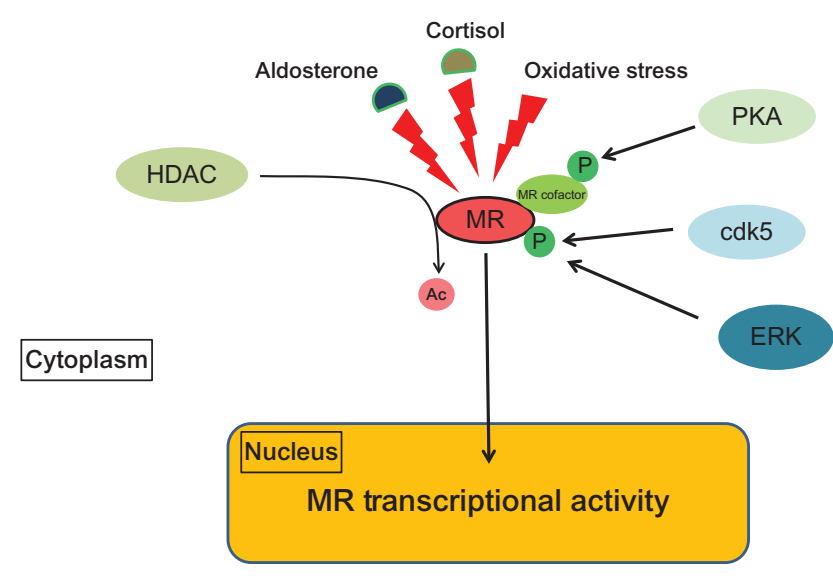

Figure 2

Potential modulation of adipocyte MR activity by post-translational modifications. The phosphorylation and acetylation state of MR affects its transcriptional activity. MR hyperacetylation leads to reduced transcriptional activity of MR. Direct phosphorylation of MR by ERK and cdk5 has been described, although the effects on the transcriptional activity are not still clear. PKA activity has also been shown to promote MR transactivation.

degradation of the receptor (Faresse et al. 2012). Such phosphorylation occurs in the ligand-bound form of MR and is prevented by ERK1/2 inhibition, suggesting that ERK can also regulate MR signaling through its direct phosphorylation (Fig. 2).

In differentiating preadipocytes, the phosphorylated form of MR is observed at day 9 of differentiation and its detection is prevented by treatment with geldanamycin, an inhibitor of Hsp90, which inhibits MR transcriptional activity (Bamberger et al. 1997, Desarzens et al. 2014). Importantly, treatment of preadipocytes with geldanamycin during differentiation impairs their conversion in mature adipose cells. These data suggest that loss of phosphorylation may alter MR transcriptional activation and, in turn, the adipogenic process. In intercalated cells of the distal nephron, phosphorylation of MR was detected at serine 843 in the ligand-binding domain and prevented ligand binding, nuclear translocation and transcriptional activity. Angiotensin II and with-no-lysine kinase 4 (WNK4) signaling inhibit dephosphorylation of this residue and promote MR activation (Shibata et al. 2013). However, the occurrence of phosphorylation at serine 843 and its effects on MR functions have never been investigated in adipose cells.

Interestingly, in cancer cells, phosphorylation of MR can also occur through cyclin-dependent kinase 5 (Cdk5), leading to reduced transcriptional activity of the receptor (Kino et al. 2010). In the adipocyte, $C d k 5$ has been shown to phosphorylate the adipogenic master regulator PPAR $\gamma$ on serine 273, with subsequent altered expression of genes encoding adiponectin and insulin-sensitizing adipokines (Choi et al. 2010). However there is still no evidence that Cdk5 is able to phosphorylate the MR directly in the adipocyte, and this aspect needs further studies.

Adipogenesis is a complex process regulated by the sequential cascade of transcription factors whose function can be modulated by the modification enzymes histone acetyltransferases and histone deacetylases (HDACs). HDACs do not only modulate the acetylation of histones but also of non-histone proteins such as DNA binding transcription factors, steroid receptors, transcription coregulators and chaperone proteins (Glozak \& Seto 2007). Recent data by Kuzmochka et al. (2014) show that inactivation of HDAC1 promotes adipose conversion. However, other studies indicate that inhibitors of HDACs repress expression of the late markers of differentiation CEBP $\alpha$ (Catalioto et al. 2009) and aP2 (Haberland et al. 2010) and curb adipogenesis. Thus, there is still controversy about the effects of HDACs activity on adipogenesis. In HEK293 cells, MR acetylation is regulated by HDAC3, and treatment with valproic acid, a HDAC inhibitor, leads to an increase in acetylation and decreased recruitment of MR on the promoter of the target gene Sgk1 (Lee et al. 2013). A similar modulation of MR activity by HDAC3 might occur in the adipocyte (Fig. 2), and affect MR ability in regulating Sgk1 and other target genes that drive adipogenesis, finally regulating adipocyte metabolism.

\section{Conclusion}

Adipocyte MR activity has been shown to regulate the expression of genes involved in adipogenesis and inflammation, both in vivo and in vitro. However, it is still unclear if MR can directly activate the transcription of target genes or if its effects are mediated through recruitment and activation of other transcription factors on the promoters of MR-regulated genes. Indirect transcriptional regulation of MR activity may include protein kinases-controlling adipogenesis, insulin sensitivity and browning of WAT; notably, modulation of MR transcriptional activity by post-translational modification of the receptor could occur, adding a further level of complexity to MR function.

In obese mouse models, treatment with MR antagonists results in several beneficial effects on the metabolic profile. At a molecular level, in the adipocyte, MR antagonism inhibits transcription of adipogenic and inflammatory genes, downregulates transcript levels of enzymes producing ROS, promotes transcription of brown

Published by Bioscientifica Ltd 
fat genes and reduces the autophagic flux. However, the molecular mechanisms downstream of the MR antagonism, which lead to the marked changes in the transcriptional profile of adipose tissue, are still largely unknown.

Indeed, a deeper understanding of the signaling pathways regulated by MR in the adipose tissue may lead to the discovery of novel molecular targets for therapies to treat obesity and its related metabolic dysfunctions.

\section{Declaration of interest}

The authors declare that there is no conflict of interest that could be perceived as prejudicing the impartiality of this review.

\section{Funding}

This work was supported by a grant from Ministero della Salute (BANDO 2011-2012 Progetti Collaborazione Ricercatori Italiani all'Estero; project grant PE-2011-02347070 to M C)

\section{Acknowledgements}

The authors are grateful to Alessandra Feraco and Caterina Mammi for critically reading the manuscript. The authors wish to dedicate this review to the memory of Sandra Meinel.

\section{References}

Armani A, Mammi C, Marzolla V, Calanchini M, Antelmi A, Rosano GM, Fabbri A \& Caprio M 2010 Cellular models for understanding adipogenesis, adipose dysfunction, and obesity. Journal of Cellular Biochemistry 110 564-572. (doi:10.1002/jcb.22598)

Armani A, Cinti F, Marzolla V, Morgan J, Cranston GA, Antelmi A, Carpinelli G, Canese R, Pagotto U \& Quarta C 2014a Mineralocorticoid receptor antagonism induces browning of white adipose tissue through impairment of autophagy and prevents adipocyte dysfunction in high-fat-diet-fed mice. FASEB Journal 28 3745-3757. (doi:10.1096/ fj.13-245415)

Armani A, Marzolla V, Rosano G \& Caprio M 2014b Mineralocorticoid vs glucocorticoid receptors: solo players or team mates in the control of adipogenesis? International Journal of Obesity 38 1580-1581. (doi:10.1038/ijo.2014.68)

Baerga R, Zhang Y, Chen PH, Goldman S \& Jin S 2009 Targeted deletion of autophagy-related 5 (atg5) impairs adipogenesis in a cellular model and in mice. Autophagy 5 1118-1130. (doi:10.4161/auto.5.8.9991)

Bamberger CM, Wald M, Bamberger AM \& Schulte HM 1997 Inhibition of mineralocorticoid and glucocorticoid receptor function by the heat shock protein 90-binding agent geldanamycin. Molecular and Cellular Endocrinology 131 233-240. (doi:10.1016/S0303-7207(97)00115-9)

Banks AS, McAllister FE, Camporez JP, Zushin PJ, Jurczak MJ, LaznikBogoslavski D, Shulman GI, Gygi SP \& Spiegelman BM 2015 An ERK/Cdk5 axis controls the diabetogenic actions of PPAR $\gamma$. Nature 517 391-395. (doi:10.1038/nature13887)

Bender SB, McGraw AP, Jaffe IZ \& Sowers JR 2013 Mineralocorticoid receptor-mediated vascular insulin resistance: an early contributor to diabetes-related vascular disease? Diabetes 62 313-319. (doi:10.2337/ db12-0905)

Bluher M, Michael MD, Peroni OD, Ueki K, Carter N, Kahn BB \& Kahn CR 2002 Adipose tissue selective insulin receptor knockout protects against obesity and obesity-related glucose intolerance. Developmental Cell $\mathbf{3}$ 25-38. (doi:10.1016/S1534-5807(02)00199-5)

Bost F, Aouadi M, Caron L, Even P, Belmonte N, Prot M, Dani C, Hofman P, Pagès G \& Pouysségur J 2005 The extracellular signal-regulated kinase isoform ERK1 is specifically required for in vitro and in vivo adipogenesis. Diabetes 54 402-411. (doi:10.2337/diabetes.54.2.402)

Boulton TG \& Cobb MH 1991 Identification of multiple extracellular signal-regulated kinases (ERKs) with antipeptide antibodies. Cell Regulation 2 357-371.

Caprio M, Feve B, Claes A, Viengchareun S, Lombes M \& Zennaro MC 2007 Pivotal role of the mineralocorticoid receptor in corticosteroid-induced adipogenesis. FASEB Journal 21 2185-2194. (doi:10.1096/fj.067970com)

Caprio M, Antelmi A, Chetrite G, Muscat A, Mammi C, Marzolla V, Fabbri A, Zennaro MC \& Feve B 2011 Antiadipogenic effects of the mineralocorticoid receptor antagonist drospirenone: potential implications for the treatment of metabolic syndrome. Endocrinology 152 113-125. (doi:10.1210/en.2010-0674)

Carlson CJ, Koterski S, Sciotti RJ, Poccard GB \& Rondinone CM 2003 Enhanced basal activation of mitogen-activated protein kinases in adipocytes from type 2 diabetes: potential role of p38 in the downregulation of GLUT4 expression. Diabetes 52 634-641. (doi:10.2337/diabetes.52.3.634)

Catalioto RM, Maggi CA \& Giuliani S 2009 Chemically distinct HDAC inhibitors prevent adipose conversion of subcutaneous human white preadipocytes at an early stage of the differentiation program. Experimental Cell Research 315 3267-3280. (doi:10.1016/j.yexcr.2009. 09.012)

Cho HJ, Park J, Lee HW, Lee YS \& Kim JB 2004 Regulation of adipocyte differentiation and insulin action with rapamycin. Biochemical and Biophysical Research Communications 321 942-948. (doi:10.1016/j.bbrc. 2004.07.050)

Choi JH, Banks AS, Estall JL, Kajimura S, Boström P, Laznik D, Ruas JL, Chalmers MJ, Kamenecka TM, Blüher M et al. 2010 Anti-diabetic drugs inhibit obesity-linked phosphorylation of PPAR $\gamma$ by Cdk5. Nature 466 451-456. (doi:10.1038/nature09291)

Cinti S 2012 The adipose organ at a glance. Disease Models \& Mechanisms 5 588-594. (doi:10.1242/dmm.009662)

De Kloet ER, Van Acker SA, Sibug RM, Oitzl MS, Meijer OC, Rahmouni K \& de Jong W 2000 Brain mineralocorticoid receptors and centrally regulated functions. Kidney International 57 1329-1336. (doi:10.1046/j. 1523-1755.2000.00971.x)

Desarzens S, Liao WH, Mammi C, Caprio M \& Faresse N 2014 Hsp90 blockers inhibit adipocyte differentiation and fat mass accumulation. PLoS ONE 9 e94127. (doi:10.1371/journal.pone.0094127)

Di Pietro N, Panel V, Hayes S, Bagattin A, Meruvu S, Pandolfi A, Hugendubler L, Fejes-Tóth G, Naray-Fejes-Tóth A \& Mueller E 2010 Serum- and glucocorticoid-inducible kinase 1 (SGK1) regulates adipocyte differentiation via forkhead box O1. Molecular Endocrinology 24 370-380. (doi:10.1210/me.2009-0265)

Doorn J, Leusink M, Groen N, van de Peppel J, van Leeuwen JP, van Blitterswijk CA \& de Boer J 2012 Diverse effects of cyclic AMP variants on osteogenic and adipogenic differentiation of human mesenchymal stromal cells. Tissue Engineering. Part A 18 1431-1442. (doi:10.1089/ten. tea.2011.0484)

Ebisuya M, Kondoh K \& Nishida E 2005 The duration, magnitude and compartmentalization of ERK MAP kinase activity: mechanisms for providing signaling specificity. Journal of Cell Science 118 2997-3002. (doi:10.1242/jcs.02505)

Faresse N 2014 Post-translational modifications of the mineralocorticoid receptor: How to dress the receptor according to the circumstances? Journal of Steroid Biochemistry and Molecular Biology 143 334-342. (doi:10.1016/j.jsbmb.2014.04.015)

Faresse N, Vitagliano JJ \& Staub O 2012 Differential ubiquitylation of the mineralocorticoid receptor is regulated by phosphorylation. FASEB Journal 26 4373-4382. (doi:10.1096/fj.12-209924) 
Farman N \& Bocchi B 2000 Mineralocorticoid selectivity: molecular and cellular aspects. Kidney International 57 1364-1369. (doi:10.1046/j. 1523-1755.2000.00976.x)

Funder JW 2000 Aldosterone and mineralocorticoid receptors: orphan questions. Kidney International 57 1358-1363. (doi:10.1046/j.15231755.2000.00975.x)

Funder JW 2005 Mineralocorticoid receptors: distribution and activation. Heart Failure Reviews 10 15-22. (doi:10.1007/s10741-005-2344-2)

Funder JW 2009 Reconsidering the roles of the mineralocorticoid receptor. Hypertension 53 286-290. (doi:10.1161/HYPERTENSIONAHA.108. 119966)

Furukawa S, Fujita T, Shimabukuro M, Iwaki M, Yamada Y, Nakajima Y, Nakayama O, Makishima M, Matsuda M \& Shimomura I 2004 Increased oxidative stress in obesity and its impact on metabolic syndrome. Journal of Clinical Investigation 114 1752-1761. (doi:10.1172/JCI21625)

Gagnon A, Chen CS \& Sorisky A 1999 Activation of protein kinase B and induction of adipogenesis by insulin in 3T3L1 preadipocytes: contribution of phosphoinositide-3,4,5-trisphosphate versus phosphoinositide-3,4-bisphosphate. Diabetes 48 691-698. (doi:10.2337/diabetes.48.4.691)

Galigniana MD 1998 Native rat kidney mineralocorticoid receptor is a phosphoprotein whose transformation to a DNA-binding form is induced by phosphatases. Biochemical Journal 333 555-563. (doi:10.1042/bj3330555)

Glick D, Barth S \& Macleod KF 2010 Autophagy: cellular and molecular mechanisms. Journal of Pathology 221 3-12. (doi:10.1002/path.2697)

Glozak MA \& Seto E 2007 Histone deacetylases and cancer. Oncogene 26 5420-5432. (doi:10.1038/sj.onc.1210610)

Guo C, Ricchiuti V, Lian BQ, Yao TM, Coutinho P, Romero JR, Li J, Williams GH \& Adler GK 2008 Mineralocorticoid receptor blockade reverses obesity-related changes in expression of adiponectin, peroxisome proliferator-activated receptor- $\gamma$, and proinflammatory adipokines. Circulation 117 2253-2261. (doi:10.1161/CIRCULATIONAHA.107.748640)

Haberland M, Carrer M, Mokalled MH, Montgomery RL \& Olson EN 2010 Redundant control of adipogenesis by histone deacetylases 1 and 2 . Journal of Biological Chemistry 285 14663-14670. (doi:10.1074/jbc. M109.081679)

Harms M \& Seale P 2013 Brown and beige fat: development, function and therapeutic potential. Nature Medicine 19 1252-1263. (doi:10.1038/ nm.3361)

Hirata A, Maeda N, Hiuge A, Hibuse T, Fujita K, Okada T, Kihara S, Funahashi T \& Shimomura I 2009 Blockade of mineralocorticoid receptor reverses adipocyte dysfunction and insulin resistance in obese mice. Cardiovascular Research 84 164-172. (doi:10.1093/cvr/cvp191)

Hoppmann J, Perwitz N, Meier B, Fasshauer M, Hadaschik D, Lehnert H \& Klein J 2010 The balance between gluco- and mineralo-corticoid action critically determines inflammatory adipocyte responses. Journal of Endocrinology 204 153-164. (doi:10.1677/JOE-09-0292)

Kajimura S, Seale P \& Spiegelman BM 2010 Transcriptional control of brown fat development. Cell Metabolism 11 257-262. (doi:10.1016/ j.cmet.2010.03.005)

Kino T, Jaffe H, Amin ND, Chakrabarti M, Zheng YL, Chrousos GP \& Pant HC 2010 Cyclin-dependent kinase 5 modulates the transcriptional activity of the mineralocorticoid receptor and regulates expression of brain-derived neurotrophic factor. Molecular Endocrinology 24 941-952. (doi:10.1210/me.2009-0395)

Kovsan J, Blüher M, Tarnovscki T, Klöting N, Kirshtein B, Madar L, Shai I, Golan R, Harman-Boehm I, Schön MR et al. 2011 Altered autophagy in human adipose tissues in obesity. Journal of Clinical Endocrinology and Metabolism 96 E268-E277. (doi:10.1210/jc.2010-1681)

Kuhn E, Bourgeois C, Keo V, Viengchareun S, Muscat A, Meduri G, Le MD, Feve B \& Lombes M 2014 Paradoxical resistance to high-fat diet-induced obesity and altered macrophage polarization in mineralocorticoid receptor-overexpressing mice. American Journal of Physiology. Endocrinology and Metabolism 306 E75-E90. (doi:10.1152/ajpendo.00323.2013)
Kuzmochka C, Abdou HS, Hache RJ \& Atlas E 2014 Inactivation of histone deacetylase 1 (HDAC1) but not HDAC2 is required for the glucocorticoid-dependent CCAAT/enhancer-binding protein $\alpha$ $(\mathrm{C} / \mathrm{EBP} \alpha)$ expression and preadipocyte differentiation. Endocrinology 155 4762-4773. (doi:10.1210/en.2014-1565)

Lee HA, Lee DY, Cho HM, Kim SY, Iwasaki Y \& Kim IK 2013 Histone deacetylase inhibition attenuates transcriptional activity of mineralocorticoid receptor through its acetylation and prevents development of hypertension. Circulation Research 112 1004-1012. (doi:10.1161/CIRCRESAHA.113.301071)

Li F, Wang D, Zhou Y, Zhou B, Yang Y, Chen H \& Song J 2008 Protein kinase A suppresses the differentiation of 3T3L1 preadipocytes. Cell Research 18 311-323. (doi:10.1038/cr.2008.12)

Lin YH, Chou CH, Wu XM, Chang YY, Hung CS, Chen YH, Tzeng YL, Wu VC, Ho YL, Hsieh FJ et al. 2014 Aldosterone induced galectin-3 secretion in vitro and in vivo: from cells to humans. PLoS ONE 9 e95254. (doi:10.1371/journal.pone.0095254)

Martinez-Lopez N, Athonvarangkul D, Sahu S, Coletto L, Zong H, Bastie CC, Pessin JE, Schwartz GJ \& Singh R 2013 Autophagy in Myf5+ progenitors regulates energy and glucose homeostasis through control of brown fat and skeletal muscle development. EMBO Reports $\mathbf{1 4}$ 795-803. (doi:10.1038/embor.2013.111)

Marzolla V, Armani A, Feraco A, De Martino MU, Fabbri A, Rosano G \& Caprio M 2014 Mineralocorticoid receptor in adipocytes and macrophages: a promising target to fight metabolic syndrome. Steroids 91 46-53. (doi:10.1016/j.steroids.2014.05.001)

Massaad C, Houard N, Lombes M \& Barouki R 1999 Modulation of human mineralocorticoid receptor function by protein kinase A. Molecular Endocrinology 13 57-65. (doi:10.1210/mend.13.1.0226)

McCormick JA, Bhalla V, Pao AC \& Pearce D 2005 SGK1: a rapid aldosterone-induced regulator of renal sodium reabsorption. Physiology 20 134-139. (doi:10.1152/physiol.00053.2004)

Mizushima N 2007 Autophagy: process and function. Genes and Development 21 2861-2873. (doi:10.1101/gad.1599207)

Mizushima N \& Levine B 2010 Autophagy in mammalian development and differentiation. Nature Cell Biology 12 823-830. (doi:10.1038/ ncb0910-823)

Murano I, Barbatelli G, Parisani V, Latini C, Muzzonigro G, Castellucci M \& Cinti S 2008 Dead adipocytes, detected as crown-like structures, are prevalent in visceral fat depots of genetically obese mice. Journal of Lipid Research 49 1562-1568. (doi:10.1194/jlr.M800019-JLR200)

Nagai Y, Miyata K, Sun GP, Rahman M, Kimura S, Miyatake A, Kiyomoto H, Kohno M, Abe Y, Yoshizumi M et al. 2005 Aldosterone stimulates collagen gene expression and synthesis via activation of ERK1/2 in rat renal fibroblasts. Hypertension 46 1039-1045. (doi:10.1161/01.HYP. 0000174593.88899.68)

Nakae J, Kitamura T, Kitamura Y, Biggs WH III, Arden KC \& Accili D 2003 The forkhead transcription factor Foxo1 regulates adipocyte differentiation. Developmental Cell 4 119-129. (doi:10.1016/S1534-5807(02)00401-X)

Novikoff AB, Novikoff PM, Rosen OM \& Rubin CS 1980 Organelle relationships in cultured 3T3L1 preadipocytes. Journal of Cell Biology 87 180-196. (doi:10.1083/jcb.87.1.180)

Pearson G, Robinson F, Beers GT, Xu BE, Karandikar M, Berman K \& Cobb MH 2001 Mitogen-activated protein (MAP) kinase pathways: regulation and physiological functions. Endocrine Reviews 22 153-183.

Reul JM \& De Kloet ER 1985 Two receptor systems for corticosterone in rat brain: microdistribution and differential occupation. Endocrinology 117 2505-2511. (doi:10.1210/endo-117-6-2505)

Reusch JE, Colton LA \& Klemm DJ 2000 CREB activation induces adipogenesis in 3T3L1 cells. Molecular and Cellular Biology 20 1008-1020. (doi:10.1128/MCB.20.3.1008-1020.2000)

Rickard AJ, Morgan J, Tesch G, Funder JW, Fuller PJ \& Young MJ 2009 Deletion of mineralocorticoid receptors from macrophages protects against deoxycorticosterone/salt-induced cardiac fibrosis and increased blood pressure. Hypertension 54 537-543. (doi:10.1161/HYPERTENSIONAHA.109.131110) 
Rondinone CM, Rodbard D \& Baker ME 1993 Aldosterone stimulated differentiation of mouse 3T3L1 cells into adipocytes. Endocrinology 132 2421-2426

Rosenwald M, Perdikari A, Rulicke T \& Wolfrum C 2013 Bi-directional interconversion of brite and white adipocytes. Nature Cell Biology $\mathbf{1 5}$ 659-667. (doi:10.1038/ncb2740)

Seale P, Bjork B, Yang W, Kajimura S, Chin S, Kuang S, Scimè A, Devarakonda S, Conroe HM, Erdjument-Bromage H et al. 2008 PRDM16 controls a brown fat/skeletal muscle switch. Nature 454 961-967. (doi:10.1038/nature07182)

Seale P, Kajimura S \& Spiegelman BM 2009 Transcriptional control of brown adipocyte development and physiological function - of mice and men. Genes and Development 23 788-797. (doi:10.1101/gad.1779209)

Shibata S, Rinehart J, Zhang J, Moeckel G, Castaneda-Bueno M, Stiegler AL, Boggon TJ, Gamba G \& Lifton RP 2013 Mineralocorticoid receptor phosphorylation regulates ligand binding and renal response to volume depletion and hyperkalemia. Cell Metabolism 18 660-671. (doi:10.1016/j.cmet.2013.10.005)

Singh R, Xiang Y, Wang Y, Baikati K, Cuervo AM, Luu YK, Tang Y, Pessin JE, Schwartz GJ, Czaja MJ et al. 2009 Autophagy regulates adipose mass and differentiation in mice. Journal of Clinical Investigation 119 3329-3339. (doi:10.1172/JCI35541)

Taniguchi CM, Emanuelli B \& Kahn CR 2006 Critical nodes in signalling pathways: insights into insulin action. Nature Reviews. Molecular Cell Biology 7 85-96. (doi:10.1038/nrm1837)

Usher MG, Duan SZ, Ivaschenko CY, Frieler RA, Berger S, Schutz G, Lumeng CN \& Mortensen RM 2010 Myeloid mineralocorticoid receptor controls macrophage polarization and cardiovascular hypertrophy and remodeling in mice. Journal of Clinical Investigation 120 3350-3364. (doi:10.1172/JCI41080)

Viengchareun S, Penfornis P, Zennaro MC \& Lombes M 2001 Mineralocorticoid and glucocorticoid receptors inhibit UCP expression and function in brown adipocytes. American Journal of Physiology. Endocrinology and Metabolism 280 E640-E649.
Wada T, Ohshima S, Fujisawa E, Koya D, Tsuneki H \& Sasaoka T 2009 Aldosterone inhibits insulin-induced glucose uptake by degradation of insulin receptor substrate (IRS) 1 and IRS2 via a reactive oxygen speciesmediated pathway in 3T3L1 adipocytes. Endocrinology 150 1662-1669. (doi:10.1210/en.2008-1018)

Wada T, Kenmochi H, Miyashita Y, Sasaki M, Ojima M, Sasahara M, Koya D, Tsuneki H \& Sasaoka T 2010 Spironolactone improves glucose and lipid metabolism by ameliorating hepatic steatosis and inflammation and suppressing enhanced gluconeogenesis induced by high-fat and highfructose diet. Endocrinology 151 2040-2049. (doi:10.1210/en.2009-0869)

Wu J, Boström P, Sparks LM, Ye L, Choi JH, Giang AH, Khandekar M, Virtanen KA, Nuutila P, Schaart G et al. 2012 Beige adipocytes are a distinct type of thermogenic fat cell in mouse and human. Cell $\mathbf{1 5 0}$ 366-376. (doi:10.1016/j.cell.2012.05.016)

Wu J, Cohen P \& Spiegelman BM 2013 Adaptive thermogenesis in adipocytes: is beige the new brown? Genes and Development 27 234-250. (doi:10.1101/gad.211649.112)

Yang J \& Young MJ 2009 The mineralocorticoid receptor and its coregulators. Journal of Molecular Endocrinology 43 53-64. (doi:10.1677/ JME-09-0031)

Yang DC, Tsay HJ, Lin SY, Chiou SH, Li MJ, Chang TJ \& Hung SC 2008 cAMP/PKA regulates osteogenesis, adipogenesis and ratio of RANKL/OPG mRNA expression in mesenchymal stem cells by suppressing leptin. PLoS ONE 3 e1540. (doi:10.1371/journal.pone.0001540)

Zennaro MC, Le MD, Viengchareun S, Walker F, Ricquier D \& Lombes M 1998 Hibernoma development in transgenic mice identifies brown adipose tissue as a novel target of aldosterone action.. Journal of Clinical Investigation 101 1254-1260. (doi:10.1172/JCI1915)

Zhang JW, Klemm DJ, Vinson C \& Lane MD 2004 Role of CREB in transcriptional regulation of CCAAT/enhancer-binding protein $\beta$ gene during adipogenesis. Journal of Biological Chemistry 279 4471-4478. (doi:10.1074/jbc.M311327200)

Zhang Y, Goldman S, Baerga R, Zhao Y, Komatsu M \& Jin S 2009 Adiposespecific deletion of autophagy-related gene 7 (atg7) in mice reveals a role in adipogenesis. PNAS 106 19860-19865. (doi:10.1073/pnas.0906048106)

Received in final form 6 August 2015

Accepted 13 August 2015

Accepted Preprint published online 13 August 2015
(C) 2015 Society for Endocrinology Printed in Great Britain 